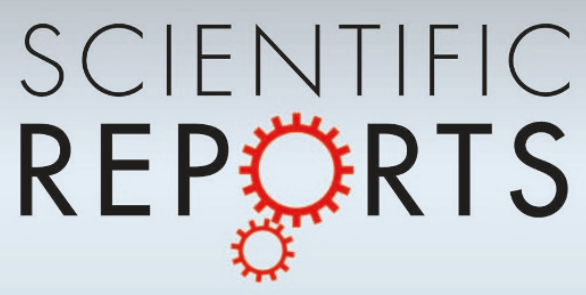

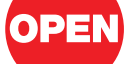

SUBJECT AREAS:

NANOSCALE BIOPHYSICS

BIOMATERIALS-CELLS

TISSUE ENGINEERING AND REGENERATIVE MEDICINE

IMPLANTS

Received

3 December 2012

Accepted

17 December 2012

Published

25 January 2013

Correspondence and requests for materials should be addressed to M.C. (marco. cecchini@nano.cnr.it) or R.B. (r.bizzarri@sns.

\title{
Unveiling LOX-1 receptor interplay with nanotopography: mechanotransduction and atherosclerosis onset
}

\author{
Carmine Di Rienzo ${ }^{1,2}$, Emanuela Jacchetti ${ }^{1}$, Francesco Cardarelli ${ }^{2}$, Ranieri Bizzarri' ${ }^{1}$, \\ Fabio Beltram' \& Marco Cecchini'
}

${ }^{1}$ NEST, Istituto Nanoscienze-CNR and Scuola Normale Superiore; Piazza San Silvestro 12, 56127 Pisa (ITALY), ${ }^{2}$ Center for Nanotechnology Innovation @ NEST, Istituto Italiano di Tecnologia, Piazza San Silvestro 12, 1-56127 Pisa, Italy.

Lectin-like ox-LDL receptors (LOX-1) play a crucial role in the ox-LDL-induced pathological transformation of vessel-wall components, a crucial early step in atherogenesis. LOX-1 dynamics is quantitatively investigated in human endothelial cells (HUVECs) exposed to environmental nanotopographies. We demonstrate distinct nanotopography-induced cell phenotypes, characterized by different morphology, LOX-1 diffusivity and oligomerization state: HUVECs on flat surfaces exhibit the behavior found in pro-atherogenic conditions, while growth on nanogratings can interfere with LOX-1 dynamics and lead to a behavior characteristic of normal, non-pathological conditions.

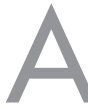

therosclerosis occurs in large and medium-size arteries where lesions containing lipids, protein and death cells can form ${ }^{1}$. The appearance of atherosclerotic plaques in defined spatial regions suggests a local inclination towards pro-atherosclerotic pathways concomitant to a diffuse activation of anti-atherosclerotic pathways ${ }^{2}$. Oxidized low density lipoproteins (ox-LDLs) are considered among the most effective activators of the pro-atherosclerotic pathways and were linked to atherogenesis and plaque rupture ${ }^{1,3}$. In this context, the endothelial lectin-like ox-LDL receptor (LOX-1) plays a crucial role in the ox-LDL-induced pathological transformation of vessel-wall components, a crucial early step in atherogenesis ${ }^{4,5}$. A number of investigations have linked LOX-1 to atherogenesis. It was shown, for instance, that mice lacking LOX-1 against a background of null LDL receptors exhibit significantly reduced atherosclerotic plaque formation compared to mice harboring LOX-1 in similar background conditions ${ }^{6}$. By contrast, mice overexpressing LOX-1 against an apolipoprotein $\mathrm{E}(\mathrm{apoE})$ deficient background exhibit an enhanced atherosclerotic plaque formation ${ }^{7}$. These observations indicate that ox-LDL binding to LOX-1 accelerates formation and progression of atherosclerotic plaques.

From a molecular point of view, it is worth noting that well-known chemical stimuli involved in atherogenesis are also able to regulate LOX-1 expression on the cell membrane ${ }^{8-13}$. In this context, it has been recently reported that the LOX-1 dimer is necessary for efficient ox-LDL uptake. Furthermore, the dimerization process is concentration-dependent and it does not need ox-LDL stimulation ${ }^{14-16}$. Thus, LOX-1 is expressed at low concentration and kept monomeric in a normal endothelium, while it is overexpressed and starts to aggregate during the early stages of atherosclerosis ${ }^{17,18}$. The recurrent disposition of atherosclerotic plaques (i.e. close to arterial bifurcations) suggests that their formation results from a highly regulated interplay between homogenously distributed chemical stimuli (e.g. ox-LDL) and localized pro-atherosclerotic events, as it has been demonstrated in detail for physical stresses ${ }^{19}$. Here, cytoskeleton-mediated and integrins-mediated mechanotransduction clearly emerges as one of the main driving forces in endothelial dysfunction at the earliest steps in atherogenesis $^{2,20,21}$. The effectiveness of mechanotransduction depends on several external regulators, including shear stress, cell-cell interactions, and cell-matrix interactions ${ }^{19,22-25}$

In spite of these findings, no one has yet adequately described the correlation between the extracellular matrix topographic features and atherogenesis onset. Indeed, ECM topography stands as one of the most relevant modulators of cell physiology and provides a "non-chemical" activation pathway to tune cell functionality/ properties ${ }^{26-28}$. Accordingly, in this work artificial scaffolds with controlled nano-/micro-topography were applied to investigate how cell-ECM interactions influence LOX-1 behaviour. More specifically, we report on LOX-1 receptor dynamics and aggregation on the cell membrane and its interaction with the cytoskeleton and the extracellular environment in different topographic contexts. Notably we used a GFP-tagged variant of LOX-129 

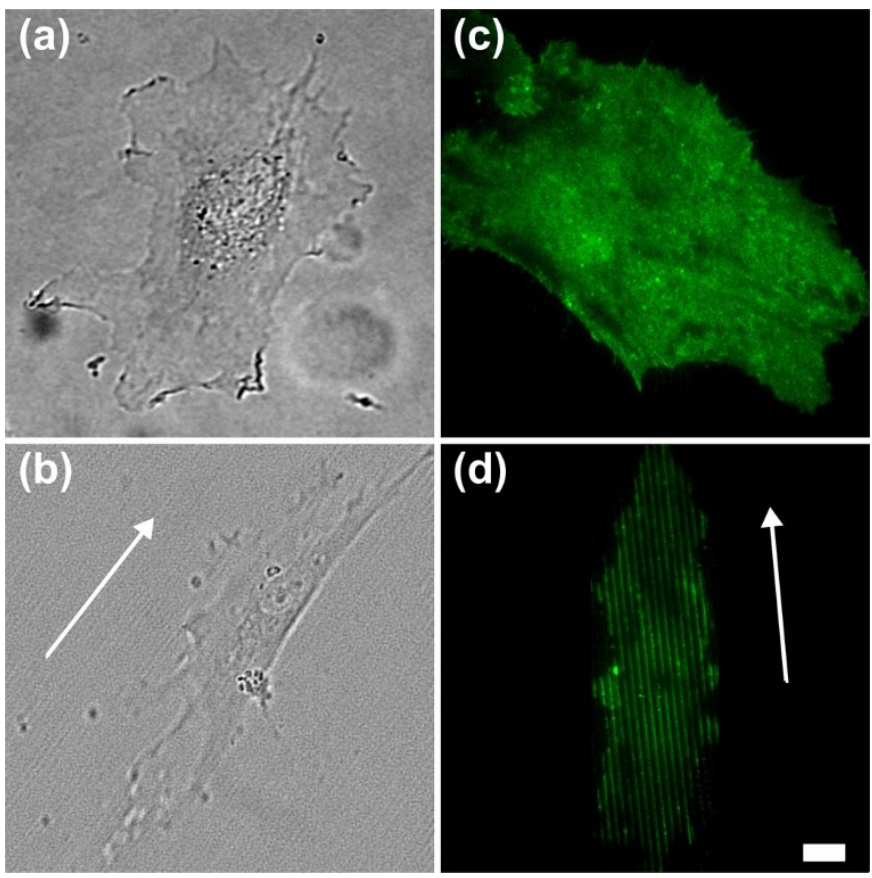

Figure $1 \mid$ Effect of topography on HUVEC morphology and LOX-1 spatial distribution. Representative bright field images of HUVECs adhered on (a) FLAT and (b) nanogratings. Representative TIRFM images of LOX-1-GFP transfected HUVECs on (c) FLAT and (d) nanogratings. Scale bar $10 \mu \mathrm{m}$. White arrows indicate the substrate directionality.

that can be transiently transfected into human endothelial cells (HUVECs) and is not activated by external ligands, thus providing the ideal model to study the sole influence of cytoskeletal factors and external topography on the receptor mobility and aggregation. Receptor dynamics was quantitatively investigated by combining high-resolution fluorescence microscopy, fluorescence correlation techniques, and micro-/nano-fabrication methodologies. First, we tested the effects of mechano-topographical stimuli on LOX-1 diffusivity in cells using an original approach that combines advanced fluorescence correlation techniques ${ }^{30,31}$ and micro-/nano-fabrication. Next, we investigated the relation between cellular function/ structure and LOX-1 diffusivity by treating cells with cytoskeletondisrupting agents. Finally, we linked our results on LOX-1 diffusion in various topographic conditions to its oligomerization state in living cells based on a fluctuation spectroscopic technique named number and brightness $(\mathrm{N} \& \mathrm{~B})$ analysis ${ }^{32}$. Our results clearly demonstrate the existence of distinct topography-induced endothelial phenotypes, characterized by different cell morphology, reactive-oxygenspecie (ROS) production, LOX-1 diffusivity and oligomerization state. Most importantly, the data allow us to identify peculiar pro-/ anti-atherosclerotic conditions that might take place during atherosclerosis onset.

\section{Results}

Morphology of HUVECs on nanostructured surfaces. In the first set of experiments, we studied the morphology of HUVECs on FLAT and patterned surfaces. In order to determine suitable substrate geometry for efficient HUVEC mechanotransduction, we initially assayed nanogratings of three different periods, namely $1 \mu \mathrm{m}(\mathrm{T} 1)$, $2 \mu \mathrm{m}$ (T2) and $4 \mu \mathrm{m}$ (T4) (see Materials and Methods for definitions and characteristics). Figure 1 and Suppl. Fig. 2 provide representative images of HUVECs attached on FLAT, T1, T2 and T4. On FLAT, cells were found to spread without polarization and showed the typical star-like shape (Fig. 1 a). By contrast, on patterned substrates they showed polarized morphology, with the cell stretched along a preferential axis that follows the substrate directionality (Fig. $1 \mathrm{~b}$ and Suppl. Fig. $2 \mathrm{a}-\mathrm{b}$ ). In order to quantify the polarization on the different employed geometries (Suppl. Fig. 1), we measured cell spreading (adhesion area), cell elongation (L) and cell alignment to substrate directionality $(\varphi)$ (Fig. 2 and Suppl. Fig. 3). The mean adhesion area of HUVECs on FLAT substrates is $1600 \pm 200 \mu^{2}$, in good agreement with literature data ${ }^{37}$. Conversely, HUVECs covered T1 samples with a significantly reduced area compared to the FLAT surfaces (40\%, Suppl. Fig. 3 a). Significantly, on longer-period topographies (T2, T4) cells showed spreading areas similar to FLAT surfaces (Fig. 2 and Suppl. Fig. 2 a). As previously stated, HUVECs showed increased elongation along the pattern direction on T1, T2, and T4, compared to FLAT values (Suppl. Fig. $3 \mathrm{~b}$ and $\mathrm{c}$ ). Maximum elongation was detected on the $\mathrm{T} 2$ substrates $(\mathrm{L}=2.2 \pm 0.2$ compared to $\mathrm{L}=1.0 \pm$ 0.1 on FLAT, Fig. 2 b) together with optimal alignment to substrate directionality $\left(\varphi=10.0^{\circ} \pm 0.5^{\circ}\right.$, Fig. 2 c). Taken together, these data demonstrate that $\mathrm{T} 2$ performed best during HUVEC spreading and contact guidance. For this reason, we selected T2 for the following experiments, and kept the FLAT as an anisotropic control condition.

We tested how non-physiological conditions associated with specific cytoskeletal degradation affect the morphology of HUVECs attached to flat or patterned substrates. First we investigated the effect of microtubule disruption by administrating nocodazole (NCZ). On T2 NCZtreated cells showed a reduction in alignment from $\varphi=10.0^{\circ} \pm 0.5^{\circ}$ (non-treated cells) to $\varphi=16^{\circ} \pm 2^{\circ}$ (Suppl. Fig. $4 \mathrm{c}$ ), and, in elongation, from $\mathrm{L}=2.2 \pm 0.2$ (non-treated cells) to $\mathrm{L}=1.1 \pm 0.1$ (Suppl. Fig. $4 \mathrm{~b}$ ). Notably, NCZ-treated cells showed a reduced elongation factor with all substrates (Suppl. Fig. 4 b). Then, we tested the effect of actin-filament disruption on polarization. Cytochalasin D-treated cells showed negligible variations of elongation and alignment, although a detectable reduction (about 40\%) of the adhesion area on both T2 and FLAT substrates was observed (Suppl. Fig. 3 a). Finally, we tested the contractility depletion effect by treating HUVECs with blebbistatin. Treated cells showed no significant difference in spreading area on FLAT or T2 (Fig. 2 (a)). On the other hand, we measured strongly reduced elongation on T2 surface compared to the physiological state (Fig. 2 (b)) which led to values comparable to the cell elongation on FLAT. At the same time we observed reduced alignment between the cells and the pattern (Fig. 2 (c)).

LOX-1-GFP localization and diffusivity. In a further set of experiments, we investigated how adhesion on FLAT or T2 modulates localization and single-molecule mobility of LOX-1-GFP. Singlemolecule mobility was assessed by fluorescence correlation spectroscopy (FCS) measurements. Average membrane mobility of the receptor in each point was determined by correlating images of a temporal stack, according to the time image correlation technique $(\mathrm{tICS})^{31}$. Line-scanning FCS was applied to determine the directional mobility of the receptor between two given points in a segment ${ }^{30}$. Additionally, the extent of autocorrelation at each point along the lines allowed estimating the relative concentration values, since correlation amplitude and molecular concentration are inversely proportional ${ }^{38}$.

Figure $1 \mathrm{c}$ and $\mathrm{d}$ shows representative images of HUVECs transiently transfected with GFP-tagged LOX-1 on FLAT and T2 substrates, respectively. LOX-1-GFP distribution is uniform on FLAT. By contrast, a non-uniform membrane distribution can be clearly seen on the patterned substrates (further evidence of this effect is also reported in Suppl. Fig. 2 for other geometries). In particular, 'ridge' regions are always characterized by significantly higher fluorescence intensity compared to 'groove' regions, as detected by TIRF and confocal imaging. Line-scanning FCS allowed attributing the higher fluorescence to higher LOX-1-GFP concentration on the ridges (Suppl. Fig. 3). Indeed, correlation amplitudes in grooves were always higher than on the ridges. 
Next, we measured average LOX-1-GFP diffusivity by tICS. By fitting tICS data to a $2 \mathrm{D}$ model of diffusion suitable for membrane studies $^{39,40}$, it was found that $\mathrm{D}=0.081 \pm 0.004 \mu \mathrm{m}^{2} / \mathrm{s}$ on FLAT (Fig.2 (d)), a value in agreement with those displayed by membrane proteins of similar size $e^{41,42}$. Remarkably, on T2 we measured an almost twofold increase in the diffusivity of LOX-1-GFP [D=0.143 $\pm 0.006 \mu \mathrm{m}^{2} / \mathrm{s}$, Fig. 2(d)]. In order to strengthen and support our analysis, we also performed the same experiment on $\mathrm{T} 1$ and $\mathrm{T} 4$, obtaining similar values (Suppl. Fig. 3d).

Upon NCZ treatment, we found $\mathrm{D}=0.124 \pm 0.006 \mu \mathrm{m}^{2} / \mathrm{s}$ on $\mathrm{T} 2$, and $\mathrm{D}=0.082 \pm 0.004 \mu \mathrm{m}^{2} / \mathrm{s}$ on FLAT (Suppl. Fig. $3 \mathrm{~d}$ ). This slight reduction of diffusivity on T2 substrates compared to the physiological state has no high statistical significance. The same trend was observed upon Cytochalasin D treatment $\left(\mathrm{D}=0.124 \pm 0.007 \mu \mathrm{m}^{2} / \mathrm{s}\right.$ on $\mathrm{T} 2$, and $\mathrm{D}=0.08 \pm 0.01 \mu \mathrm{m}^{2} / \mathrm{s}$ on FLAT, Suppl. Fig. $3 \mathrm{~d}$ ). Thus, the membrane mobility of LOX-1-GFP was found to be unaffected by microtubule and actin depolymerization both on FLAT and patterned surfaces. Conversely, the mobility of LOX-1-GFP on T2 was significantly hampered $\left(D=0.10 \pm 0.01 \mu \mathrm{m}^{2} / \mathrm{s}\right.$, Fig. $\left.2 \mathrm{~d}\right)$ by blebbistatin and became statistically indistinguishable from FLAT values $\left(D=0.10 \pm 0.02 \mu \mathrm{m}^{2} / \mathrm{s}\right.$, Fig. $\left.2 \mathrm{~d}\right)$. This suggests a key role of contractility in the topography-induced modulation of LOX-1-GFP mobility.

In order to further investigate the influence of patterned substrates on the membrane mobility of LOX-1-GFP, we adopted the pair correlation function (pCF) approach to line $\mathrm{FCS}^{30,43}$. Indeed, by analyzing the spatial correlation of fluorescence fluctuations at a pair of points in each sample separated by a distance comparable to the topography period, we were able to probe the single-molecule mobility and directionality of LOX-1-GFP membrane traffic with respect to the substrate structure. All experiments were carried out at physiological conditions: a representative pCF measurement is reported in Fig. 3. For simplicity, we focused on pair correlations along two directions: perpendicular (blue arrow, Fig. 3a) and parallel (red arrow, Fig. 3a) to the substrate pattern directionality. For T2, the
pCF calculated at the distance of $1 \mu \mathrm{m}$ parallel to the substrate directionality (red curve, Fig. 3b) yields a correlation peak earlier in time with respect to that calculated orthogonally (blue curve, Fig. 3b). This indicates that LOX-1-GFP moves faster along ridges and grooves than across them. This effect was quantified by the mobility ratio $\mathrm{R}$ (ratio between the time of CF peaks relevant to parallel and orthogonal directions to pattern, respectively). For $\mathrm{T} 2, \mathrm{R}=1.8 \pm 0.8$, indicating a significant anisotropy in LOX-1-GFP mobility on patterned substrates. As a control, the same analysis was repeated on FLAT (Fig. 3c): the pCFs calculated in two orthogonal directions yielded correlation maxima with no clear difference in their timing. This suggests that there is no preferential route taken by LOX-1-GFP in HUVECs adhered on FLAT. Accordingly, we found a mean mobility ratio $\mathrm{R}=1.0 \pm 0.3$ for FLAT. This result reinforces the idea that LOX1 -GFP mobility is significantly influenced by the spatial distribution of ridges and grooves that are present in the patterned surfaces.

LOX-1-GFP aggregation state in living cells. It is by now widely accepted that LOX-1 homodimerization is needed for ox-LDL internalization in living cells ${ }^{14}$, and that its oligomerization state may be locally regulated by receptor density ${ }^{15}$. The data presented so far pointed out detectable differences between LOX-1-GFP behavior on FLAT as compared with nanogratings. To investigate whether this may be related to its oligomerization state we performed the number and brightness analysis $(\mathrm{N} \& \mathrm{~B})^{32}$ on stacks of fluorescence images. The results obtained are displayed in Fig. 4. As a reference for 'monomeric' molecular brightness on the membrane we transiently transfected HUVECs with a farnesyl-conjugated $\mathrm{GFP}^{39}$ (Fig. 4 a, central panel). As expected we found a homogeneous distribution of the monomer on the membrane (Fig. 4 a, left panel, points in the green rectangle) with a corresponding average molecular brightness $\left(\varepsilon_{\text {monomer }}\right)$ of $0.066 \pm 0.003$ counts/ molecule/pixel-dwell-time. This value was taken as reference for analogous measurements on cells transiently transfected with LOX-1-GFP on FLAT and T2 substrates (Fig. $4 \mathrm{e}-\mathrm{f}$ and $4 \mathrm{~h}-\mathrm{i}$ ). (a)

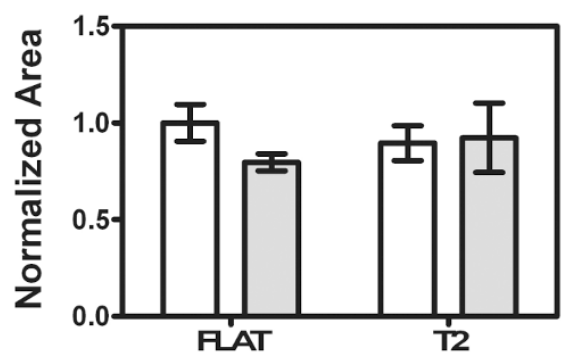

(c)

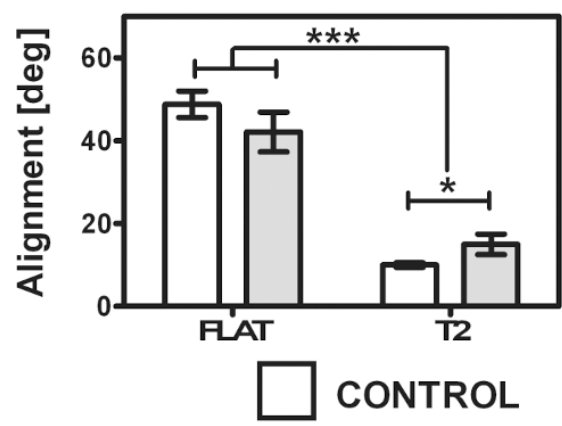

(b)

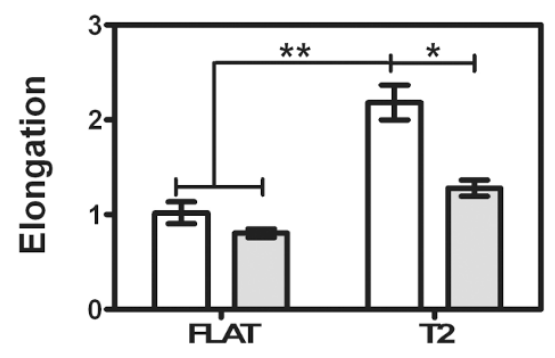

(d)

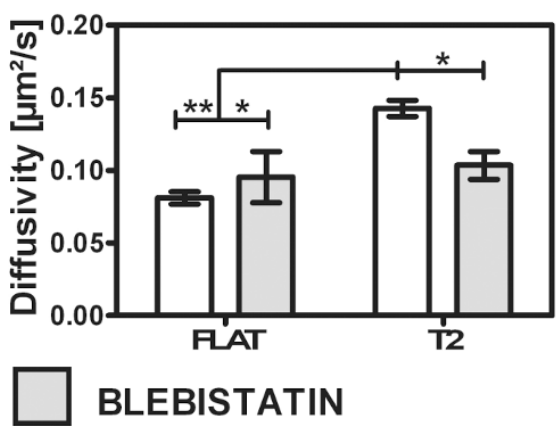

Figure $2 \mid$ Effect of contractility inhibition on HUVECs Morphology and LOX-1 diffusivity on FLAT and T2 substrates. The histograms report (a) the adhesion area, (b) elongation and (c) alignment of LOX-1 transfected cells, and (d) LOX-1 diffusivity. Experiments were performed with and without blebbistatin administration. Data are reported as mean \pm standard error of 4 experiments. 275 cells were analyzed. Significant differences between the population means are reported. 
(a)

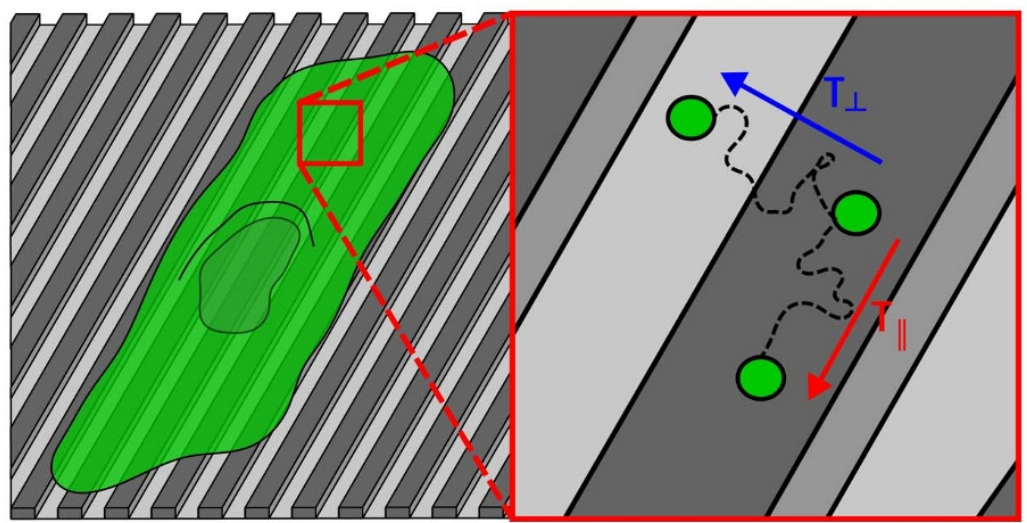

(b)

(c)
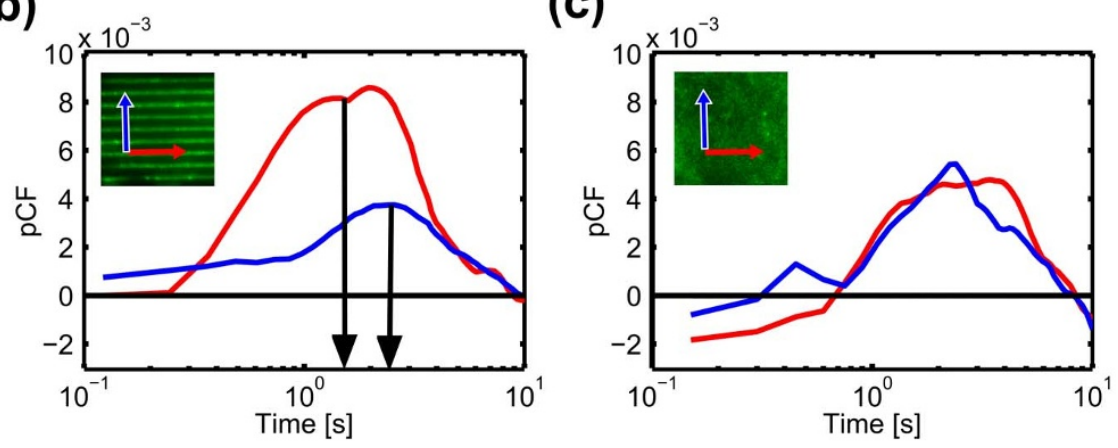

Figure $3 \mid$ Anisotropic LOX-1 diffusion. (a) Scheme of LOX-1-GFP-transit-time measure where $\tau_{\|}$is the LOX-1 mean time to cross $1 \mu \mathrm{m}$ along the pattern direction and $\tau_{\perp}$ is the same coefficient along the orthogonal one. (b-c) Representative LOX-1 pCF along the two directions (parallel red curve, perpendicular blue curve) of a single HUVEC cell adhered on (b) T2 and (c) FLAT substrates. The insets represent the two analyzed directions.

In line with the literature, N\&B analysis of LOX-1-GFP on FLAT substrates shows a coexistence of monomers and dimers, resulting in a mean molecular brightness of $0.102 \pm 0.008$, approximately 1.5 times higher than the monomeric reference value. This result suggests that LOX-1-GFP transfection yields membrane protein concentration values above the dimerization threshold (Fig.4 e-f). Remarkably, a similar analysis demonstrates that the dimeric population almost completely disappears when cells are plated on T2 substrates, showing a concomitant increase of the monomeric form fraction $(\varepsilon=0.070 \pm 0.004$, Fig. $4 \mathrm{~g}-\mathrm{i})$.

Reactive oxygen specie (ROS) production of HUVECs on nanostructured surfaces. In order to study the effect of nanotopography on HUVEC oxidative stress, cytoplasmic ROS concentration was measured for cells grown on T2 and FLAT. Using a combination of two dyes and fluorescence microscopy (TBHP and $\mathrm{c}-\mathrm{H}_{2}$ DCFDA, see Materials and Methods for details), oxidative stress could be quantitatively evaluated. Regardless of the presence of nanopatterning, our substrates did not induce significant stress in HUVECs. Indeed, ROS concentration on FLAT was reduced to one-third with respect to the positive control induced by TBHP. Nonetheless, we found that cells on T2 showed a marked decrease $(44 \pm 10 \%, \mathrm{p}<0.05$, Mann-Whitney $U$ test $)$ in ROS production with respect to cells on the FLAT. This indicates that the presence of nanopatterning can lead to a less stressful condition for endothelial cells than standard flat surfaces.

\section{Discussion}

There is strong evidence for a role of oxidative stress in all stages of atherosclerosis $^{44,45}$. Oxidized low density lipoprotein (ox-LDL), a marker of oxidative stress, is present in the plasma and in the atherosclerotic arteries of patients with atherosclerosis. Ox-LDL leads to endothelial activation, dysfunction and injury ${ }^{46}$. Recently, a novel lectin-like scavenger receptor for ox-LDL (LOX-1) was identified, primarily in the endothelial vascular smooth muscle, lymphoid cells (including macrophages) and platelets, which allows the uptake of ox-LDL and cell activation ${ }^{4,47-49}$. Also, recent transgenic animal model studies show that LOX-1 plays a significant role in atherosclerotic plaque initiation and progression ${ }^{6,7,50}$. Administration of LOX-1 antibodies in cellular and animal models consistently suggests that such intervention inhibits atherosclerosis ${ }^{51}$. Despite its important role in the process of atherogenesis, the most effective means of targeting and disrupting OxLDL-LOX-1 endocytosis remains elusive. Promisingly, Ishigaki et al showed that LOX-1 expressed ectopically in the liver via adenovirus administration reduces the levels of circulating OxLDL and inhibits the formation of atherosclerotic lesions ${ }^{52}$. Gene therapy with possible genomic manipulation of scavenger receptor expression by delivery of transgenes or by blockade of gene expression was also suggested ${ }^{53}$. In our opinion, such complex in vivo approaches can be usefully supported by in vitro studies, where many molecular details of LOX-1 activity relevant to pathogenesis (e.g. diffusion, oligomerization state, etc.) can be isolated and studied in detail at the single-molecule level. To this aim we expressed a GFP-labeled variant of LOX-1 in live human endothelial cells and studied its behavior by means of highresolution/high sensitivity fluorescence microscopy. This variant of LOX-1 is unable to bind to ox-LDL although it retains all features of native LOX-1 and it is therefore the ideal system to visualize the sole effect of ECM topography on LOX-1-dependent cell dysfunction.

Experimentally, a toolbox of correlation-based techniques was used to quantitatively address LOX-1-GFP local diffusivity (by tICS), spatial heterogeneity of flow (by pair-correlation) and oligomerization state (by Number and Brightness analysis). At the same time, by micro-/nano-fabrication methodologies we prepared 

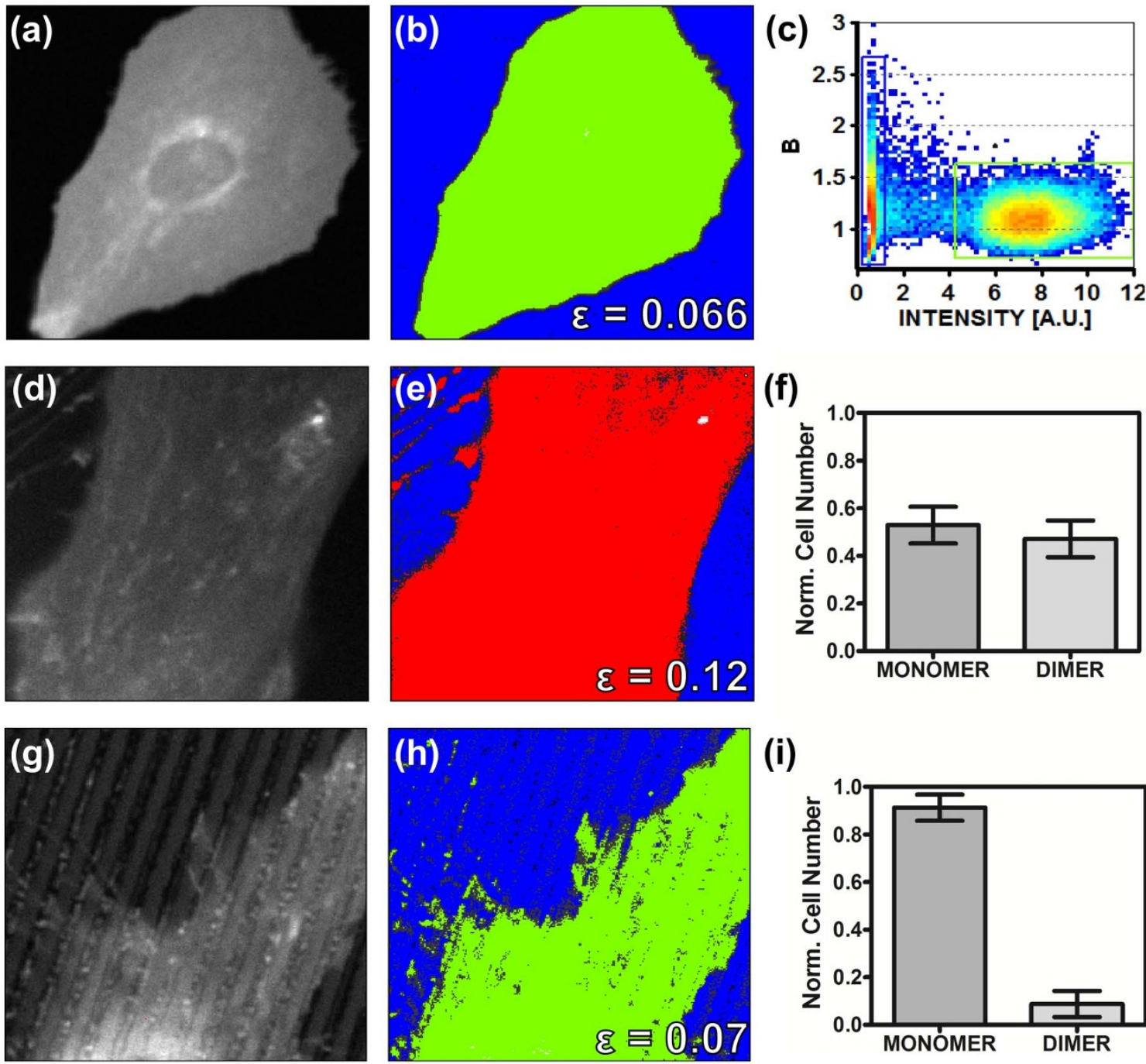

(i)

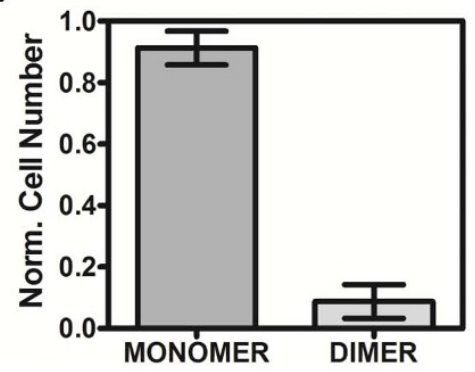

Figure 4 | LOX-1 aggregation state. (a-c) Calibration of monomeric GFP brightness using farnesyl-GFP. (a) fluorescence intensity image of a cell expressing farnesyl-GFP shows widespread distribution of the protein on the membrane (b) Selection of pixels with brightness corresponding to the monomers (green, $\mathrm{B}=1.066, \varepsilon=0.066$ ) and to the background (blue) (c) brightness vs intensity plot showing the "green" and "blue" selections. (d-f) Representative N\&B analysis of a HUVEC cell on FLAT with LOX-1 in its dimeric form. (d) intensity image (e) Selection of pixels with brightness corresponding to the dimers (red, $\mathrm{B}=1.12, \varepsilon=0.12$ ) and to background (blue) (f) cumulative results: percentage of cells showing the LOX-1 monomer (and dimer) on FLAT. (g-i) Representative N\&B analysis of a HUVEC cell on T2 with LOX-1 in its monomeric form (g) intensity image (h) Selection of pixels with brightness corresponding to the monomers (green, $\mathrm{B}=1.07, \varepsilon=0.07$ ) and to the background (blue). Cumulative results: in this case about the $90 \%$ of the analyzed cells indicates the presence of the LOX-1 monomer only.

controlled-pattern substrates allowing investigating different environmental conditions for cell attachment, spreading, and growth. By this integrated approach we demonstrated the interplay between cell spreading/polarization, LOX-1 dynamics/oligomerization state, and LOX-1 interactions with the cytoskeleton and with the extracellular environment. In particular, as schematized in Fig. 5, we identified two distinct conditions: $i$ ) when placed in isotropic environments (cells cultured on flat surfaces) LOX-1 is evenly split into a homodimeric and monomeric form, it is homogenously distributed within the membrane wherein it diffuses isotropically, and, $i$ ) when in an anisotropic environment (polarized cells adhered on nanogratings) LOX-1 shows preferential accumulation in regions of higher adhesion (ridge) and is preferentially locked in its monomeric form throughout the membrane. The monomeric form of LOX-1 displays higher diffusivity values that are dependent on contractility and topography. It is worth mentioning that topography orientation drives the main orientation of the cytoskeleton components. This in turn suggests that such ordered cellular structures may play a role in directing LOX-1 diffusion in membranes. Accordingly, both contractility depletion and flat substrates are able to abolish the observed anisotropic increase in LOX-1 diffusivity. In this context, a recent work on the CD36 macrophage scavenger receptor demonstrates the existence of different diffusive behaviors, such as isotropic diffusion and linear diffusion, "guided" by cytoskeleton elements. The latter motion, interestingly, confers a higher effective diffusivity to the receptor and correlates to its functional aggregation state ${ }^{42}$.

It is well known that LOX-1 homodimerization is required for receptor activation and subsequent ox-LDL internalization ${ }^{14}$. Based on this and on the results discussed above, the endothelial cells adhered on flat substrates can be considered more atherogenesisprone then endothelial cells adhered on nanogratings (e.g. T2) where LOX-1 is locked in an inactive form (Fig. 6). In our results, cells with similar LOX-1 expression levels yield remarkable differences in LOX-1 aggregation/activation state as a function of the substrate used. Overall, these results suggest that it is possible to regulate the LOX-1 oligomerization state (and then its disposition to internalize 


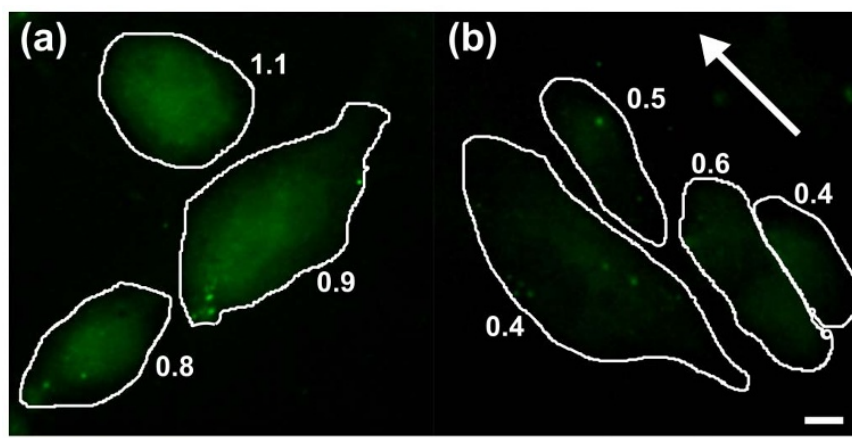

(c)

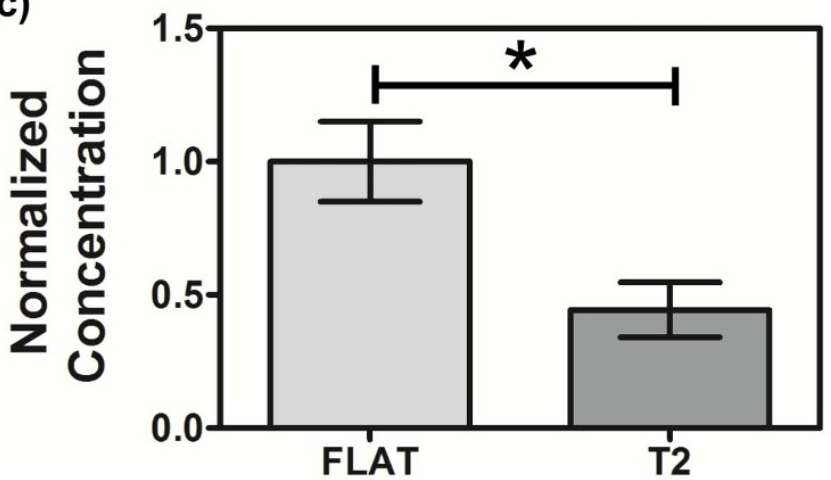

Figure 5 $\mid$ ROS production in different topographical condition. Representative fluorescence images of cells treated with $\mathrm{c}-\mathrm{H}_{2}$ DCFDA on FLAT (a) and T2 (b), respectively. Cell contours are shown for clarity. The white arrow indicates substrate directionality. Scale bar $10 \mu \mathrm{m}$. (c) Normalized average ROS concentration in HUVECs on FLAT and T2. 651 cells were analyzed. Data are reported as mean \pm standard error of 3 experiments.

ox-LDL) manipulating the cellular environment. Generation of ROS is inevitable for aerobic organisms ${ }^{54}$, but healthy cells maintain a controlled rate of ROS levels. An increase can be correlated to an alteration of membrane lipids, proteins, and nucleic acids and is also associated with a wide variety of pathological events including atherosclerosis ${ }^{55}$. Basal oxidative stress could be modulated by chemical environment but also by the ECM topography and, in particular, our results show that $\mathrm{T} 2$ nanograting is able to reduce the endothelial cell oxidative stress. Along this reasoning, the two ideal conditions tested here (periodic grating vs smooth surface) may be related, respectively, to the non-atherosclerotic intima, where endothelial cells are exposed to an ordered mesh of collagen fibers, and to the aged/atherosclerotic tissue, where the same cells are instead exposed to a continuous deposition of amorphous substances, such as elastin and collagen ${ }^{56-58}$.

We believe that these results should be taken into account for the rational engineering of stents for cardiovascular surgery. Among the drawbacks limiting their more widespread use is the long-term endothelial dysfunction and inability to adapt to growth ${ }^{58}$. Many studies demonstrate that topographical features modulate surface endothelialization and endothelial integration with fluxes ${ }^{37,59}$. Our results contribute to this picture by isolating and investigating the role of nanotopographical modulation on protein activity in endothelial dysfunction and atherosclerosis.

\section{Methods}

Substrate fabrication. Nanogratings (alternating lines of grooves and ridges with submicrometer lateral dimension) were fabricated by thermal nano imprint lithography (NIL) as previously described ${ }^{33}$. Copolymer 2-norbornene ethylene [cyclic olefin copolymer (COC)] foils (IBIDI. Martinsried, Germany) was selected as thermoplastic material because of favorable biocompatibility and optical properties ${ }^{34}$.

The imprinting process comprises 5 steps: (1) the substrate is placed between the mold and a glass cover slip inside the NIL system; (2) the temperature is raised to $150^{\circ} \mathrm{C}$; (3) 50 -bar-pressure is applied for $5 \mathrm{~min}$; (4) the system is cooled to $70^{\circ} \mathrm{C}$; (5) finally, the pressure is released. The imprinted foils are removed from the NIL system, detached from mold and then attached by using silicone glue (RS Components, Italy) to the bottom of hollowed $35 \mathrm{~mm}$ Petri dishes. We produced nanogratings characterized by periods of $1 \mu \mathrm{m}$ (T1), $2 \mu \mathrm{m}$ (T2) and $4 \mu \mathrm{m}$ (T4), and depth of $350 \mathrm{~nm}$.

Before each experiment, the substrates were sterilized in ethanol for $15 \mathrm{~min}$, and then washed twice in PBS and in culture medium.

Cell culture, transfection and treatments. Low-passage human umbilical vein endothelial cells (HUVECs) were grown in M200 medium supplemented with $2 \% \mathrm{v} / \mathrm{v}$ fetal bovine serum (FBS), $1 \mu \mathrm{g} / \mathrm{ml}$ hydrocortisone, $10 \mathrm{ng} / \mathrm{ml}$ human epidermal growth factor (EGF), $3 \mathrm{ng} / \mathrm{ml}$ basic fibroblast growth factor (FGF), and $10 \mu \mathrm{g} / \mathrm{ml}$ heparin (all reagents from Invitrogen, Carlsbad, USA) and maintained at $37^{\circ} \mathrm{C}$ and $5 \% \mathrm{CO}_{2}$.

Cells were seeded at a density of $2 \times 10^{4} \mathrm{cell} / \mathrm{s} / \mathrm{cm}^{2}$ and culture medium was replaced 24 hours after seeding to remove residuals. The GFP-tagged variant of LOX-1 was

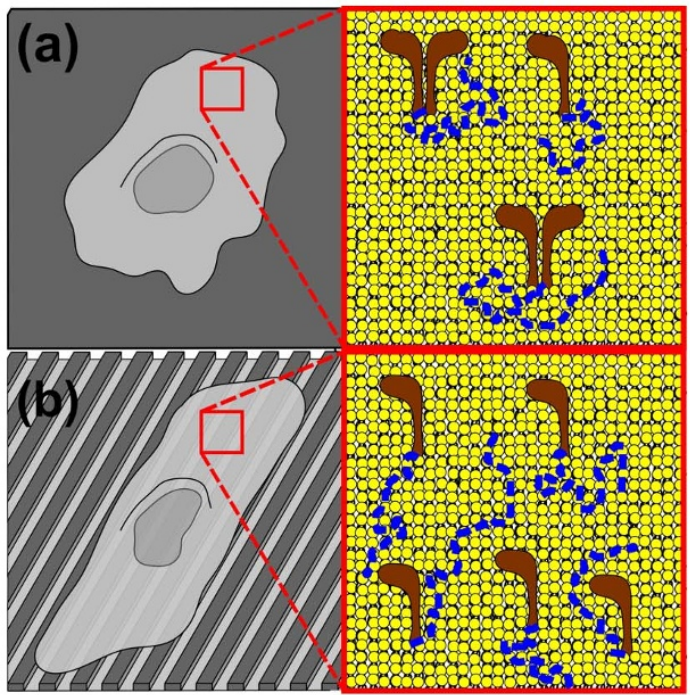

\section{Proatherogenic}

Cell polarization

LOX-1 mobility

LOX-1 Aggregation State

ROS

\section{Prophysiological}

Cell polarization

LOX-1 mobility

LOX-1 Aggregation State

ROS

Figure 6 | Pro-atherogenic and pro-physiological topographical environments. (a) HUVECs on flat surfaces adhere in a not polarized way. In this condition LOX-1 has a dimeric form and diffuses more slowly in membrane. It is active in ox-LDL binding and internalization, disposing HUVECs to a proatherogenic condition. (b) HUVECs on nanogratings adhere in a polarized way, showing a stretched and aligned morphology. LOX-1 diffuses faster and in a contractility-dependent way; furthermore it is blocked in a monomeric form that is considered unable to bind and internalize ox-LDL, protecting HUVECs against ox-LDL induced atherogenesis. 
transiently transfected using electroporation (Neon, Digital Bio), according to manufacturer's instructions.

For microtubule and actin-filament disassembly experiments, cells were treated with $50 \mathrm{nM}$ nocodazole (NCZ) and $1 \mu \mathrm{M}$ cytochalasin $\mathrm{D}$, respectively, for $15 \mathrm{~min}$ before washing with fresh medium and starting the measurements. For contractility inhibition experiments, cells were treated for $30 \mathrm{~min}$ with $50 \mu \mathrm{M}$ blebbistatin before washing with fresh medium and starting the measurements (reagents from Sigma-Aldrich, USA).

Morphology analysis. Images of the basal membrane acquired for tICS analysis (see below) were also used for morphology analysis. Cell area and orientation angle were obtained using the "Area" and "Feret's diameter" tools of ImageJ, respectively. Adhesion area results were normalized to the FLAT corresponding values. The substrate directionality angle was subtracted from the cell orientation angle: the range of possible cell/substrate alignment angles ' $\varphi$ ' varied between $0^{\circ}$ and $90^{\circ}$. The "Feret's diameter" tool also provides the major ("Feret") and minor ("MinFeret") cell axis lengths: these values were used to quantify the cell elongation factor 'L', defined as $\mathrm{L}=[($ Feret $/$ MinFeret $)-1]$.

Reactive-Oxygen-Specie (ROS) detection. HUVECs were treated with $1 \mu \mathrm{M}$ carboxy-H2DCFDA (5-(and-6)-carboxy-2', $7^{\prime}$-dichlorodihydrofluorescein diacetate ) solution (Molecular Probes, Milan, Italy) at $37^{\circ} \mathrm{C}$ while protected from light. After 30 min the samples were washed in PBS and cell culture medium was added. Ten images were acquired in bright field and fluorescence by using a Nikon Ti-eclipse microscope equipped with an immersion-oil 40x objective (NA 1.3). Tert-butyl hydroperoxide (TBHP, Invitrogen, Milan, Italy) was used for the positive control ${ }^{35}$. The fluorescence background was subtracted from each image. At least 100 cells were analysed per experiment. Single cell profiles were manually traced by using the bright-field images and the average fluorescence for each ROI was then calculated. Results were normalized to the fluorescence value measured for FLAT.

Temporal Image Correlation Spectroscopy (tICS) and pair Correlation Function (pCF) analysis. tICS and pCF measurements were carried out with a Leica AF6000 fluorescence microscope in total internal reflection mode (TIRFM), with a penetration depth of the evanescent wave of less than $100 \mathrm{~nm}$. Images were acquired by illuminating the sample with a $488 \mathrm{~nm}$ laser while using a 100x (NA 1.47) oil-immersion objective to collect fluorescence and a cooled EM-CCD Hamamatsu C1900-13 for recording. For each cell between 800 to 2000 frames were collected with a frequency of 8 to $12 \mathrm{~Hz}$. tICS was used to quantify the diffusion coefficient (D, $\mu \mathrm{m}^{2} / \mathrm{s}$ ) of the protein in each pixel of an image series. For each cell, membrane pixels were manually selected and autocorrelation functions were calculated using previously published Matlab code ${ }^{31}$, which were then fitted in the Matlab environment. The pair correlation function $p C F(n)$ is a cross-correlation function of the fluorescence time-trace at two different points that differ by a distance of ' $n$ ' $\operatorname{pixels}^{30}$. The $p C F(n)$ function at a given pixel distance ' $n$ ' is given by the following expression:

$$
p C F(n)=G(\tau, \delta r)=\frac{\langle F(t, 0) \cdot F(t+\tau, \delta r)\rangle}{\langle F(t, 0)\rangle\langle F(t, \delta r)\rangle}-1
$$

where $F(t, n)$ is the fluorescence intensity at time $t$ at pixel $n$ along the scan line, $\tau$ is the time shift and $\delta r$ is the distance between pixels. In the present experiments the distance between adjacent pixels is $228 \mathrm{~nm}$. We used the maximum of the pCF(n) to determine the average time a molecule takes to travel a given distance. This time is different if there is an obstacle or barrier along the line of measurement. Here we typically correlate pairs of points at a distance of $1 \mu \mathrm{m}$ both parallel and perpendicular to the substrate directionality. By contrast, in the case of the FLAT substrate, these two directions are arbitrarily chosen with respect to the cell morphology. The pCF peak positions and heights are estimated by interpolating the correlation curve with a 4-degree polynomial function. The obtained values are then used for the calculation of the mobility ratio $\mathrm{R}$, defined as:

$$
R=\frac{\tau_{\perp}}{\tau_{\|}}
$$

where $\tau_{\perp}$ and $\tau_{\|}$indicate the time position of the $\mathrm{pCF}(\mathrm{n})$ peak in the direction perpendicular and parallel to the substrate directionality, respectively. Thus $\mathrm{R}=1$ indicates that molecules need the same time to travel in the two chosen directions (as expected for isotropic diffusion). Instead, $\mathrm{R}<1$, indicates that molecules move faster in the direction parallel to the substrate directionality.

Number and Brightness (N\&B) analysis. The N\&B experiments were carried out with an Olympus FluoView 1000-ASW-2.0 confocal laser scanning microscope using a 63x (NA 1.4) planApo oil-immersion objective. The pinhole was set to 1 Airy Unit. We set the microscope to the pseudo-photon counting mode of data acquisition. In this mode, the parameters needed for $\mathrm{N} \& \mathrm{~B}$ analysis are the detector offset, the factor $\mathrm{S}$ that converts photon counts to digital levels and the readout variance $\sigma_{0}{ }^{2}$. For the analysis of this work, the values of these parameters were calibrated according to the principles described in Ref. ${ }^{36}$. We obtained and used $\mathrm{S}=3.5, \sigma_{0}{ }^{2}=0$, and offset $=0$ for all experiments. $256 \times 256$ images at 12 bits were collected with a pixel dwell time of $40 \mu \mathrm{s}$. A time series of 150 frames with no programmed delay between images was used to reduce statistical error. Low laser power was chosen to avoid photobleaching. The N\&B analysis was performed using the SimFCS software (www.lfd.uci.edu). To calibrate the laser power and scanning conditions required for the measurement of a monomeric protein diffusing within membranes, we measured the brightness of HUVECs transiently expressing the non-aggregating farnesyl-EGFP adduct (EGFP-F). These conditions were then used for the LOX-1-GFP experiments.

Statistical analyses. All the values reported are mean \pm standard error of at least 3 independent experiments. In order to test if the observed population of values for each measured parameter belongs to the same distribution we performed a Mann-Whitney U test using the GraphPad Prism (GraphPad Software) commercial software. We reject the null hypothesis if $\mathrm{p}<0.05$. In these cases, '***', '**', and '*' indicate $\mathrm{p}<0.001, \mathrm{p}<0.01$ and $\mathrm{p}<0.05$, respectively.

1. Ross, R. Atherosclerosis - An Inflammatory Disease. New England Journal of Medicine 340, 115-126 (1999).

2. Hahn, C. \& Schwartz, M. A. Mechanotransduction in vascular physiology and atherogenesis. Nature reviews. Molecular cell biology 10, 53-62 (2009).

3. Glass, C. K. \& Witztum, J. L. Atherosclerosis. Cell 104, 503-516 (2001).

4. Sawamura, T. et al. An endothelial receptor for oxidized low-density lipoprotein. Nature 386, 73-7 (1997).

5. Cheng, C. et al. The Role of Shear Stress in Atherosclerosis: Action Through Gene Expression and Inflammation? Cell Biochemistry and Biophysics 41, 279-294 (2004).

6. Mehta, J. L. et al. Deletion of LOX-1 reduces atherogenesis in LDLR knockout mice fed high cholesterol diet. Circulation research 100, 1634-42 (2007).

7. Inoue, K., Arai, Y., Kurihara, H., Kita, T. \& Sawamura, T. Overexpression of lectin-like oxidized low-density lipoprotein receptor-1 induces intramyocardial vasculopathy in apolipoprotein E-null mice. Circulation research 97, 176-84 (2005).

8. Hermonat, P. L., Zhu, H., Cao, M. \& Mehta, J. L. LOX-1 Transcription. Cardiovascular drugs and therapy / sponsored by the International Society of Cardiovascular Pharmacotherapy 25, 393-400 (2011).

9. Kume, N. et al. Inducible expression of lectin-like oxidized LDL receptor-1 in vascular endothelial cells. Circulation research 83, 322-7 (1998).

10. Li, D. Y., Zhang, Y. C., Philips, M. I., Sawamura, T. \& Mehta, J. L. Upregulation of endothelial receptor for oxidized low-density lipoprotein (LOX-1) in cultured human coronary artery endothelial cells by angiotensin II type 1 receptor activation. Circulation research 84, 1043-9 (1999).

11. Li, L., Roumeliotis, N., Sawamura, T. \& Renier, G. C-reactive protein enhances LOX-1 expression in human aortic endothelial cells: relevance of LOX-1 to C-reactive protein-induced endothelial dysfunction. Circulation research $\mathbf{9 5}$, 877-83 (2004).

12. Morawietz, H. et al. Angiotensin II induces LOX-1, the human endothelial receptor for oxidized low-density lipoprotein. Circulation 100, 899-902 (1999).

13. Nagase, M. et al. Redox-sensitive regulation of lox-1 gene expression in vascular endothelium. Biochemical and biophysical research communications 281, 720-5 (2001)

14. Xie, Q. et al. Human lectin-like oxidized low-density lipoprotein receptor-1 functions as a dimer in living cells. DNA and cell biology 23, 111-7 (2004).

15. Matsunaga, S. et al. Lectin-like oxidized low-density lipoprotein receptor (LOX-1) functions as an oligomer and oligomerization is dependent on receptor density. Experimental cell research 313, 1203-14 (2007).

16. Ohki, I. et al. Surface plasmon resonance study on functional significance of clustered organization of lectin-like oxidized LDL receptor (LOX-1). Biochimica et biophysica acta 1814, 345-54 (2011).

17. Chen, M. et al. Increased Expression of Lectinlike Oxidized Low Density Lipoprotein Receptor-1 in Initial Atherosclerotic Lesions of Watanabe Heritable Hyperlipidemic Rabbits. Arterioscler Thromb Vasc Biol 20, 1107-1115 (2000).

18. Kataoka, H. et al. Expression of lectinlike oxidized low-density lipoprotein receptor-1 in human atherosclerotic lesions. Circulation 99, 3110-7 (1999).

19. Hahn, C. \& Schwartz, M. A. Mechanotransduction in vascular physiology and atherogenesis. Nature reviews. Molecular cell biology 10, 53-62 (2009).

20. Noma, K., Kihara, Y. \& Higashi, Y. Striking crosstalk of ROCK signaling with endothelial function. Journal of cardiology 60, 1-6 (2012).

21. Zaragoza, C., Márquez, S. \& Saura, M. Endothelial mechanosensors of shear stress as regulators of atherogenesis. Current opinion in lipidology 23, 446-52 (2012).

22. Miao, H. et al. Effects of flow patterns on the localization and expression of VE-cadherin at vascular endothelial cell junctions: in vivo and in vitro investigations. Journal of vascular research 42, 77-89 (2005).

23. Orr, A. W. et al. The subendothelial extracellular matrix modulates NF-kappaB activation by flow: a potential role in atherosclerosis. The Journal of cell biology 169, 191-202 (2005).

24. Hahn, C., Orr, A. W., Sanders, J. M., Jhaveri, K. A. \& Schwartz, M. A. The subendothelial extracellular matrix modulates JNK activation by flow. Circulation research 104, 995-1003 (2009).

25. Funk, S. D. et al. Matrix-specific protein kinase A signaling regulates p21-activated kinase activation by flow in endothelial cells. Circulation research 106, 1394-403 (2010).

26. Ferrari, A. et al. Nanotopographic control of neuronal polarity. Nano letters 11, 505-11 (2011). 
27. Chen, W. et al. Nanotopography Influences Adhesion, Spreading, and Self-Renewal of Human Embryonic Stem Cells. ACS nano (2012). doi:10.1021/ nn3004923.

28. Schvartzman, M. et al. Nanolithographic control of the spatial organization of cellular adhesion receptors at the single-molecule level. Nano letters 11, 1306-12 (2011).

29. Chen, M. \& Sawamura, T. Essential role of cytoplasmic sequences for cell-surface sorting of the lectin-like oxidized LDL receptor-1 (LOX-1). Journal of molecular and cellular cardiology 39, 553-61 (2005).

30. Digman, M. A. \& Gratton, E. Imaging barriers to diffusion by pair correlation functions. Biophysical journal 97, 665-73 (2009).

31. Kolin, D. L. \& Wiseman, P. W. Advances in image correlation spectroscopy: measuring number densities, aggregation states, and dynamics of fluorescently labeled macromolecules in cells. Cell biochemistry and biophysics 49, 141-64 (2007).

32. Digman, M. A., Dalal, R., Horwitz, A. F. \& Gratton, E. Mapping the number of molecules and brightness in the laser scanning microscope. Biophysical journal 94, 2320-32 (2008).

33. Meucci, S., Tonazzini, I., Beltram, F. \& Cecchini, M. Biocompatible noisy nanotopographies with specific directionality for controlled anisotropic cell cultures. Soft Matter 8, 1109-1119 (2012).

34. Ferrari, A. et al. Neuronal polarity selection by topography-induced focal adhesion control. Biomaterials 31, 4682-94 (2010).

35. Diaz, G., Liu, S., Isola, R., Diana, A. \& Falchi, A. M. Mitochondrial localization of reactive oxygen species by dihydrofluorescein probes. Histochemistry and cell biology 120, 319-25 (2003)

36. Ossato, G. et al. A two-step path to inclusion formation of huntingtin peptides revealed by number and brightness analysis. Biophysical journal 98, 3078-85 (2010).

37. Franco, D. et al. Control of initial endothelial spreading by topographic activation of focal adhesion kinase. Soft Matter (2011). doi:10.1039/c1sm05191a.

38. Digman, M. A. \& Gratton, E. Lessons in fluctuation correlation spectroscopy. Annual review of physical chemistry 62, 645-68 (2011).

39. Storti, B., Bizzarri, R., Cardarelli, F. \& Beltram, F. Intact microtubules preserve transient receptor potential vanilloid 1 (TRPV1) functionality through receptor binding. The Journal of biological chemistry 287, 7803-11 (2012).

40. Nohe, A., Keating, E., Fivaz, M., van der Goot, F. G. \& Petersen, N. O. Dynamics of GPI-anchored proteins on the surface of living cells. Nanomedicine: nanotechnology, biology, and medicine 2, 1-7 (2006).

41. Lenne, P.-F. et al. Dynamic molecular confinement in the plasma membrane by microdomains and the cytoskeleton meshwork. The EMBO journal 25, 3245-56 (2006).

42. Jaqaman, K. et al. Cytoskeletal Control of CD36 Diffusion Promotes Its Receptor and Signaling Function. Cell 146, 593-606 (2011).

43. Hinde, E. \& Cardarelli, F. Measuring the flow of molecules in cells. Biophysical Reviews 3, 119-129 (2011).

44. Mehta, J. L., Chen, J., Hermonat, P. L., Romeo, F. \& Novelli, G. Lectin-like, oxidized low-density lipoprotein receptor-1 (LOX-1): a critical player in the development of atherosclerosis and related disorders. Cardiovascular research 69 , 36-45 (2006).

45. Lu, J., Mitra, S., Wang, X., Khaidakov, M. \& Mehta, J. L. Oxidative stress and lectin-like ox-LDL-receptor LOX-1 in atherogenesis and tumorigenesis. Antioxidants \& redox signaling 15, 2301-33 (2011).

46. Berliner, J. A. et al. Atherosclerosis: Basic Mechanisms: Oxidation, Inflammation, and Genetics. Circulation 91, 2488-2496 (1995).

47. Draude, G., Hrboticky, N. \& Lorenz, R. L. The expression of the lectin-like oxidized low-density lipoprotein receptor (LOX-1) on human vascular smooth muscle cells and monocytes and its down-regulation by lovastatin. Biochemical pharmacology 57, 383-6 (1999).
48. Yoshida, H., Kondratenko, N., Green, S., Steinberg, D. \& Quehenberger, O. Identification of the lectin-like receptor for oxidized low-density lipoprotein in human macrophages and its potential role as a scavenger receptor. The Biochemical journal 334 (Pt 1), 9-13 (1998).

49. Chen, M. et al. Activation-dependent surface expression of LOX-1 in human platelets. Biochemical and biophysical research communications 282, 153-8 (2001).

50. $\mathrm{Hu}$, C. et al. LOX-1 deletion decreases collagen accumulation in atherosclerotic plaque in low-density lipoprotein receptor knockout mice fed a high-cholesterol diet. Cardiovascular research 79, 287-93 (2008).

51. Morawietz, H. LOX-1 and atherosclerosis: proof of concept in LOX-1-knockout mice. Circulation research 100, 1534-6 (2007).

52. Ishigaki, Y. et al. Impact of plasma oxidized low-density lipoprotein removal on atherosclerosis. Circulation 118, 75-83 (2008).

53. Stephen, S. L. et al. Scavenger receptors and their potential as therapeutic targets in the treatment of cardiovascular disease. International journal of hypertension 2010, 646929 (2010).

54. Batandier, C., Fontaine, E., Kériel, C. \& Leverve, X. M. Determination of mitochondrial reactive oxygen species: methodological aspects. Journal of cellular and molecular medicine 6, 175-87 (2002).

55. Heller, F. R., Descamps, O. \& Hondekijn, J. C. LDL oxidation: therapeutic perspectives. Atherosclerosis 137 Suppl, S25-31 (1998)

56. Tanimura, A., McGregor, D. H. \& Anderson, H. C. Calcification in atherosclerosis. I. Human studies. Journal of experimental pathology 2, 261-73 (1986)

57. Weber, G. \& Tosi, P. Observations with the scanning electron microscope on the development of cholesterol aortic atherosclerosis in the guinea-pig. Virchows Archiv A Pathologische Anatomie 353, 325-332 (1971).

58. Guagliumi, G. et al. Examination of the in vivo mechanisms of late drug-eluting stent thrombosis: findings from optical coherence tomography and intravascular ultrasound imaging. JACC. Cardiovascular interventions 5, 12-20 (2012).

59. Morgan, J. T. et al. Integration of basal topographic cues and apical shear stress in vascular endothelial cells. Biomaterials 33, 4126-35 (2012)

\section{Acknowledgments}

The authors thank Prof. Fabio Recchia and Dr. Vincezo Lionetti for fruitful discussions. This work was supported in part by the European Union Seventh Framework Programme (FP7/ 2007-2013) under grant agreement no. NMP4-LA-2009-229289 NanoII and grant agreement no. NMP3-SL-2009-229294 NanoCARD.

\section{Author contributions}

$\mathrm{MC}$ and RB conceived the experiments; EJ fabricated the nanogratings; EJ, CdR, and FC carried out the experiments; MC, RB, EJ, CdR, FC, and FB analyzed the data; all the authors wrote and reviewed the manuscript.

\section{Additional information}

Supplementary information accompanies this paper at http://www.nature.com/ scientificreports

Competing financial interests: The authors declare no competing financial interests.

License: This work is licensed under a Creative Commons

Attribution-NonCommercial-NoDerivs 3.0 Unported License. To view a copy of this license, visit http://creativecommons.org/licenses/by-nc-nd/3.0/

How to cite this article: Di Rienzo, C. et al. Unveiling LOX-1 receptor interplay with nanotopography: mechanotransduction and atherosclerosis onset. Sci. Rep. 3, 1141; DOI:10.1038/srep01141 (2013) 